\title{
La propharmacie, une formule pratique et sûre pour le patient
}

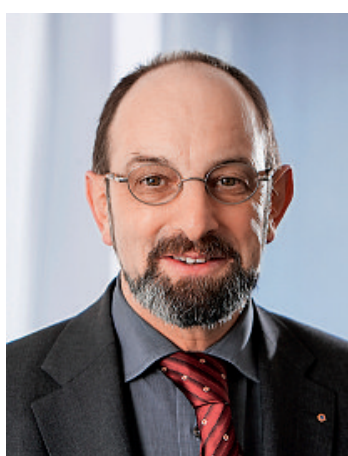

La remise de médicaments par le médecin risque d'être interdite, bien qu'elle soit pratique, sûre et avantageuse pour le patient. Dans son rapport explicatif à la consultation sur la révision de la Loi sur les produits thérapeutiques (LPTh), l'Office fédéral de la santé publique (OFSP) laisse entendre que les médecins, les dentistes et les vétérinaires ne seraient pas à même de gérer les médicaments et que leur manière actuelle de les remettre, que ce soit en urgence, au cabinet ou lors d'une visite médicale, mettrait finalement en péril la santé des patients. La remarque est osée.

L'actuelle loi sur les produits thérapeutiques a pour but de protéger la santé de l'être humain et de l'animal, ce qui lui confère un caractère de contrôle sanitaire. Quiconque souhaite interdire ou limiter la propharmacie doit tout d'abord prouver qu'elle comporte réellement des risques. L'OFSP affirme que la propharmacie engendrerait une surconsommation de médicaments néfaste pour les patients. Ces reproches sont totalement infondés. L'OFSP semble vouloir discréditer tout un groupe professionnel et lui faire porter le chapeau. Or l'argument avancé peut être facilement réfuté: si ces allégations étaient fondées, les 13 cantons qui autorisent la propharmacie devraient attester un taux de mortalité plus élevé, une espérance de vie plus faible, un nombre de maladies plus important et des coûts de médicaments par habitant nettement supérieurs. Or, c'est l'inverse qui est vrai, selon les statistiques de santésuisse, c'est justement dans ces cantons-là que les coûts des médicaments par assuré sont les plus bas.

\section{Et c'est justement dans ces cantons-là que les coûts des médicaments par assuré sont les plus bas}

Le mode de rétribution de la propharmacie nourrit aussi régulièrement le débat. Depuis des années, les médecins discutent entre eux d'une solution de rechange, et aujourd'hui de nouveau avec les assureurs. La propharmacie faisant par- tie intégrante des revenus des médecins, la valeur du point tarifaire est plus basse dans les cantons qui autorisent cette pratique. La neutralité des coûts liée à l'introduction du TARMED fait qu'un transfert sur la valeur du point tarifaire est difficilement réalisable. Le corps médical a donc soumis à santésuisse une proposition de solution actuellement en discussion.

\section{De nombreuses votations cantonales ont clairement montré que le peuple est favorable à la remise de médicaments par les médecins}

Nous autres médecins luttons avec véhémence contre une interdiction de la propharmacie, pour le bien de nos patients et pour un exercice libre de la médecine. La sécurité des médicaments et la «compliance» chez les patients sont très élevées grâce à la propharmacie: ce dont témoignent notre longue expérience auprès des patients, leur attitude face aux médicaments, leurs questions sur les différentes préparations et notamment sur les bénéfices ou les éventuels effets secondaires. Les conseils en matière de médicaments sont indissociables d'un traitement rapide, sûr, efficace, et d'une importance primordiale dans l'activité médicale. Les médecins approfondissent leurs connaissances sur les médicaments tout au long de leur carrière. Pourquoi donc enlever un outil de travail utile à ceux qui sont les mieux formés?

La propharmacie est un outil essentiel pour la mise en œuvre rapide d'un traitement sûr et efficace. Elle est garante d'une médecine de famille compétente, globale et avantageuse.

En Suisse, 4500 médecins utilisent quotidiennement cette formule pratique, sûre et de proximité, qui offre aux patients une remise des médicaments et des vaccins appropriée et de haute qualité. Les votations cantonales (AI, AR, ZH) et les décisions parlementaires dans les cantons ayant révisés leur législation en matière de santé ( $\mathrm{SZ}, \mathrm{LU}, \mathrm{UR}, \mathrm{ZG}, \mathrm{SO}, \mathrm{BL}$ ) ont clairement montré que le peuple était favorable à la remise de médicaments par les médecins.

Dr Ernst Gähler, vice-président de la FMH, Responsable du domaine Tarifs et conventions 